\title{
Evolutionary connection between C-rich AGB stars and C-rich central stars of $\mathrm{PNe}$
}

\author{
M. Parthasarathy \\ Indian Institute of Astrophysics, Bangalore - 560034, India
}

\begin{abstract}
On the basis of $\mathrm{C} / \mathrm{O}$ ratios the evolutionary connection between C-rich AGB/post-AGB stars and C-rich central stars of PNe is discussed. The proportion of carbon-rich post-AGB stars is comparable to that of carbon-rich PNe. The post-AGB stars with $21 \mu \mathrm{m}$ emission are found to be over abundant in carbon and s-process elements indicating that they have gone through the third dredge-up and carbon star phase on the AGB. As these stars evolve to higher temperature they will form carbon-rich $\mathrm{PNe}$ with carbon-rich central stars. Analysis of $\mathrm{C} / \mathrm{O}$ ratios of $\mathrm{PNe}$ suggests that 68 percent of $\mathrm{PNe}$ have $\mathrm{C} / \mathrm{O}>1$ indicating that the progenitors have gone through the third dredge-up and carbon-rich phase on the AGB. The Type I PNe can be divided into two groups. Group I Type I PNe are those with $\mathrm{C} / \mathrm{O}<1$ indicating that their massive progenitors have gone through the third dredge-up and hot bottom burning on the AGB. Group II Type I PNe are those with $\mathrm{C} / \mathrm{O}>1$. Their progenitors have gone through third dredge-up only and may not have experienced hot bottom burning. Carbon stars with silicate dust and or carbon-rich post-AGB stars (such as post-AGB stars with $21 \mu \mathrm{m}$ emission) may be the progenitors of $\mathrm{PNe}$ with [WC] central stars. The evolutionary sequence seems to be: carbon-rich AGB $\rightarrow$ carbon-rich post-AGB $\rightarrow$ [WC late type] $\rightarrow$ [WC early type] $\rightarrow$ [WC-PG 1159] $\rightarrow$ PG 1159.
\end{abstract}

\section{Introduction}

Red giant stars are believed to evolve along the asymptotic giant branch (AGB) through the $\mathrm{M} \rightarrow \mathrm{MS} \rightarrow \mathrm{S} \rightarrow \mathrm{C}$ sequence. Along this sequence the abundance of $\mathrm{C}^{12}$ increases by mixing of Helium-burning products in the stellar surface. Thus the evolution along the AGB and third dredge-up transform an oxygen-rich AGB star into a carbon-rich AGB star with overabundances of s-process elements and $\mathrm{C}^{12}$. The question of burning and mixing along the AGB is intimately linked to the carbon star mystery. Observations of the LMC and SMC prove that most of the carbon and s-process element enriched AGB stars are found at rather low luminosities indicating that they also have rather low masses $\left(1\right.$ to $\left.3 \mathrm{M}_{\odot}\right)$. On the other hand, AGB models predict the 3rd dredge-up i.e the mixing of the interior carbon to the surface during the recurrent He-shell flashes on the AGB, mostly for much higher luminosities (i.e. larger (core) masses). The reason for the absence of luminous carbon stars is most likely due to the occurrence of hot bottom burning and mass loss on the AGB. 
The hypothesis that carbon-rich AGB stars with high mass loss rates evolve into planetary nebulae (Zuckerman et al. 1976) is well supported by the result that approximately half of the mass loss from AGB stars in the solar neighbourhood results from carbon stars (Jura \& Kleinmann 1989) and that half of all planetary nebulae in the solar neighbourhood are carbon-rich (Zuckerman \& Aller 1986).

Planetary nebulae (PNe) reflect the evolutionary histories of the AGB stars that preceded them and carry the by-products of nuclear burning - enriched levels of helium, nitrogen and carbon. PNe allow to determine how the production of chemical elements depends on initial mass or metallicity and how the products are dredged-up during the late stages of stellar evolution.

Carbon abundances of post-AGB stars and PNe are of particular interest in this respect. The link between AGB stars and PNe is most directly seen from the presence of the remnants of the AGB molecular envelopes around several PNe and post-AGB stars.

From an analysis of the IRAS data several post-AGB supergiant like stars/ proto-planetary nebulae (PPNe) have been detected. The cooler $(\mathrm{K}, \mathrm{G}, \mathrm{F})$ and the hotter (A, B) post-AGB stars seem to form an evolutionary sequence in the transition region from the tip of the AGB into early stages of PNe (Parthasarathy \& Pottasch 1986; Parthasarathy 1993a, 1993b, 1994; Parthasarathy et al. 1993, 1995).

\section{Carbon-rich post-AGB stars}

The chemical composition analysis of post-AGB stars with $21 \mu \mathrm{m}$ emission shows that they are all carbon-rich and over-abundant in s-process elements. The CO and HCN data (Omont et al. 1993) and infrared spectra indicate that the dust shells around these stars are also carbon-rich. Recently Webster (1995) suggested that the $21 \mu \mathrm{m}$ emission observed in these carbon-rich post-AGB stars is due to fullerences $\mathrm{C}_{60} \mathrm{Hm}(\mathrm{m}=1$ to 60$)$. Emission at 3.3 and $3.4 \mu \mathrm{m}$, commonly attributed to polycyclic aromatic hydrocarbons (PAHs), is seen in several carbon-rich PPNe.

Recently, using mid-infrared spectra of several carbon-rich post-AGB stars with $21 \mu \mathrm{m}$ emission Justtanont et al. (1996) found in addition to the standard UIR features $(6.2,7.7,8.6,11.3 \mu \mathrm{m}$ attributed to PAHs) new features at 7.9 , $8.2,10.6,11.5$ and $12.2 \mu \mathrm{m}$, which they attribute to PAH molecule chrysene. The UIR features were also observed in several young PNe and in [WC] PNe. The $21 \mu \mathrm{m}$ feature is found only in carbon-rich post-AGB stars. There are 12 carbon-rich post-AGB stars with $21 \mu \mathrm{m}$ emission feature (Kwok 1993; Decin et al. 1998). All of them show overabundance of carbon and s-process elements indicating that they have gone through the third dredge-up and carbon star phase on the AGB (Parthasarathy et al. 1992; Hrivnak 1995; Decin et al. 1998; Reddy et al. 1997; Začs et al. 1996; Van Winckel 1997). As these stars evolve to higher temperatures they form carbon-rich PNe with carbon-rich central stars. 


\section{Carbon-rich PNe}

Zuckerman \& Aller (1986) from an analysis of $\mathrm{C} / \mathrm{O}$ ratios of large sample of $\mathrm{PNe}$ find that 62 percent of $\mathrm{PNe}$ have $\mathrm{C} / \mathrm{O}>1$. This is in agreement with the relative numbers of carbon and oxygen-rich AGB stars with large mass loss rates and is consistent with the idea that the progenitors of $\mathrm{PNe}$ are this class of AGB stars. Among PNe with [WC] type central stars about $80 \%$ percent of the nebulae are carbon-rich. Among 68 PNe studied by Zuckerman \& Aller (1986) 41 have bipolar or elliptical symmetry. Of these 59 percent are carbonrich. Therefore, bipolar or elliptical symmetry is equally probable regardless of C/O ratio. Rola \& Stasinska (1994) reexamined the problem of the discrepancy between carbon abundances in PNe derived from the CII $4267 \AA$ and CIII] 1909 $\AA$ line intensities. They find that about 50 percent of $\mathrm{PNe}$ are carbon-rich $(\mathrm{C} / \mathrm{O}$ $>1$ ). Rola and Stasinka (1994) found that 29 percent of Type I are carbonrich. Slightly lower percentage of the carbon-rich $\mathrm{PNe}$ in the sample of Rola \& Stasinka (1994) may be due to the fact that they use $\mathrm{C} / \mathrm{O}>1.2$ to consider a PN as definitely carbon-rich.

Recently Kholtygin (1998) reconsidered the problem of the difference between the abundances of carbon and oxygen in galactic planetary nebulae derived from the intensities of the recombination and collisionally excited lines. Kholtygin (1998) redetermined the $\mathrm{C} / \mathrm{O}$ ratios of a large number of $\mathrm{PNe}$. The $\mathrm{C} / \mathrm{O}$ ratios of $\mathrm{PNe}$ determined by Kholtygin are more reliable than the previous estimates. From this data we find that 68 percent of PNe are carbon-rich. Jura \& Kleinman (1989) find that about one half of all the mass losing AGB stars are carbon-rich. They conclude that the carbon star phase occurs in about one half of all main-sequence stars of 1 to $1.5 \mathrm{M}_{\odot}$.

\section{Type-I and bipolar PNe}

Their kinematic properties and galactic distribution indicate that the masses of the progenitor stars were higher than those of other PNe. Most of the galactic He- $\mathrm{N}$ rich (Type I) PNe are bipolar and most bipolar $\mathrm{PNe}$ are $\mathrm{He}-\mathrm{N}$ rich. $\mathrm{PNe}$ with $\mathrm{He} / \mathrm{H}>0.125$ or $\mathrm{N} / \mathrm{O}>0.5$ were defined as Type I (Peimbert 1978). Recently Kingsburgh \& Barlow (1994) defined Type I PNe as those with N/O ratio $(>0.8)$ higher than their initial $(\mathrm{C}+\mathrm{N}) / \mathrm{O}$ ratio implying a complete conversion of the initial $\mathrm{C}$ into $\mathrm{N}$ and also of the $\mathrm{C}$ produced by the third dredge-up.

We find that about 50 percent of Type I PNe have $\mathrm{C} / \mathrm{O}>1$ indicating that they have experienced third dredge-up. On the basis of $\mathrm{C} / \mathrm{O}$ ratios of Type $\mathrm{I}$ and bipolar PNe we find that the Type I PNe can be classified into two groups. Group I Type I PNe are those with $\mathrm{C} / \mathrm{O}<1$ and Group II Type I are those with $\mathrm{C} / \mathrm{O}>1$. These two groups may be related to the hot bottom burning and third dredge-up of progenitor stars during their evolution on the AGB. Several of the carbon-rich PNe, C-rich Type I PNe, bipolar PNe and PNe with [WC] central stars have CO molecular envelopes (Huggins et al. 1996) and molecular hydrogen emission (Kastner et al. 1996) indicating a range in masses of the progenitors for the carbon-rich $\mathrm{PNe}$.

Jura (1991) examined the published data for carbon stars losing more mass than $10^{-6} M_{\odot} \mathrm{yr}^{-1}$ in the solar neighborhood. The carbon stars with higher 
terminal velocities have significantly higher fluxes of the $\mathrm{J}=1-0$ emission line of $\mathrm{HCN}$ relative to their infrared continuum emission at $60 \mu \mathrm{m}$. Jura (1991) argued that this result is probably a reflection of a higher nitrogen abundance by a factor of 10 in these outflows. Such a high nitrogen abundance is consistent with the hypothesis that the high terminal velocity carbon stars have more massive main-sequence progenitors. It is likely that these high terminal velocity carbon stars may, themselves, be the progenitors of Type I PNe.

Recently Clegg et al. (1997) determined ${ }^{12} \mathrm{C} /{ }^{13} \mathrm{C}$ isotope ratio in the ionized gas in 2 PNe, NGC 3918 and SMC N2. Clegg et al. (1997) derived ${ }^{12} \mathrm{C} /{ }^{13} \mathrm{C}$ ratios to be 15 and 7 respectively. NGC 3918 and SMC N2 are carbon-rich (C/O 1.6 and 3.6 respectively) and have probably descended from C-rich AGB stars. The ${ }^{12} C /{ }^{13} C$ ratio in these nebulae is lower than the typical values in carbon stars. This may be due to hot bottom burning in the progenitors.

Leisy \& Dennefeld (1996) determined carbon abundances of several Type I PNe in LMC and SMC. From the study of carbon abundances in LMC and $\mathrm{SMC}$ it is found that the third dredge-up has been occurring in all PNe. The third dredge-up efficiency is higher with lower metallicities which is in agreement with the higher number of carbon stars found in metal-deficient galaxies. Leisy \& Dennefeld (1996) study of carbon abundances in LMC and SMC PNe shows that the third dredge-up carbon is sometimes transformed into nitrogen by hotbottom burning, but in a few objects only and not even in all the Type I PNe. This in agreement with our classification of Type I PNe into two groups.

Type I PNe with $\mathrm{C} / \mathrm{O}<1$ and with over-abundance of nitrogen have gone through the third dredge-up and hot-bottom burning on the AGB. They are relatively more massive than Type I PNe with $\mathrm{C} / \mathrm{O}>1$ which have probably gone through third dredge-up and a carbon-rich phase on the AGB but not experienced hot-bottom burning. The question whether the progenitors of the Type I $\mathrm{PNe}$ have gone through the carbon star stage is governed by the competition between dredge-up and hot-bottom burning, i.e. the efficiencies of both processes which, in turn, depend on convection and mass loss (see the review by Blöcker in this volume).

\section{PNe with [WC] central stars}

About 7 percent of the known central stars of planetary nebulae (CSPN) show Wolf-Rayet (WR) spectrum falling all in the [WC] class (Acker et al. 1992, 1996). The [WC] central stars are helium and carbon rich. More than 80 percent of the nebulae with $[\mathrm{WC}]$ central stars are carbon-rich $(\mathrm{C} / \mathrm{O}>1)$. The distribution of the spectral types of [WC] stars shows that most of them are either early type [WC2-4] and late type [WC9-11]. The reason for the paucity of [WC5-7] types is not clear. The temperatures of the [WC] central stars range from $20000 \mathrm{~K}$ for the [WC11] stars to as much as $150000 \mathrm{~K}$ for the [WC2-3]. The over-abundance in carbon of the PNe with [WC] central stars shows that the progenitors have gone through the third dredge-up and carbon-rich phase on the AGB. Some of the PNe with [WC] central stars are Type I and or bipolar and have CO molecular envelopes (Huggins et al. 1996) and molecular hydrogen emission (Kastner et al. 1996) indicating a range in the masses of progenitors. It is quite likely that post- 
AGB stars of different initial masses can pass through a [WC] stage with similar atmospheric parameters.

The PNe with [WC] central stars in LMC and SMC are also found to be extremely carbon-rich (Pena et al. 1997). The high electron densities of these $\mathrm{PNe}$ indicate that they are formed only recently from the termination of the AGB phase of progenitor carbon-rich stars.

The formation of [WC] central star with extreme under-abundance of hydrogen and over-abundance of $\mathrm{C}$ and $\mathrm{He}(\mathrm{C} / \mathrm{He} \simeq 1)$ may be the result of thermal pulse either at the very end of the carbon-rich AGB phase or during the young PNe stage, or when the central star is already on the white dwarf cooling track (very late thermal pulse or born-again AGB scenario, Iben 1984).

Tylenda, Acker \& Stenholm (1993) determined the [WC] subclasses for a large number of CSPN. They found several "weak emission line central stars" (WELS) which have the CIV $5805 \AA$ line systematically weaker and narrower than early [WC] CSPN and also the CIII $5696 \AA$ line very weak or absent. Parthasarathy, Acker \& Stenholm (1998) found that the characteristics of the spectra of weak emission line stars are very similar to that of [WC]-PG1159 objects. The broad HeII/CIV absorption complex some times with NLTE emission cores is the spectroscopic hallmark in the optical to identify PG1159 stars. On the basis of the spectra Parthasarathy, Acker \& Stenholm (1998) discovered 30 new [WC]-PG1159 stars. These stars mark the transition from [WC] central stars of planetary nebulae to the PG1159 (pre)white dwarfs. LM1-64 is most likely a [WC]-PG1159 CSPN in the LMC.

The presence of nebulae and dust shells around these stars and also the significant percentage of [WC]-PG1159 stars among the [WC] central stars indicate that very late thermal pulse or born-again AGB scenario is not the principal mechanism for the post-AGB evolution of [WC] stars towards the non-DA white dwarf stage.

Recent ISO observations have revealed that a significant fraction of [WC] $\mathrm{PNe}$ have cool oxygen-rich dust (Waters et al. 1998). In the wavelength region shortward of $15 \mu \mathrm{m}$ the spectra of PNe with [WC] central stars show PAH features indicating carbon-rich dust, whereas longward of $18 \mu \mathrm{m}$ solid state features from crystalline silicates indicating oxygen-rich dust are seen. The transition to [WC] type (from oxygen-rich state to carbon-rich state) may have taken place during the evolution from the tip of the AGB and before the central star attained a temperature of $20000 \mathrm{~K}$ (within a time span of the order of 1000 years).

In the infrared the spectral features of $\mathrm{PNe}$ with [WC] central stars are found to be similar to that observed in the spectra of carbon-rich post-AGB stars and post-AGB stars with $21 \mu \mathrm{m}$ emission (UIR and PAH features). Carbon stars with silicate dust and or carbon-rich post-AGB stars (such as post-AGB stars with $21 \mu \mathrm{m}$ emission) may be the progenitors of PNe with [WC] central stars. The evolutionary sequence seems to be carbon-rich AGB $\rightarrow$ carbon-rich post-AGB $\rightarrow$ [WC late $] \rightarrow[$ WC early $] \rightarrow[$ WC]-PG1159 $\rightarrow$ PG1159. 


\section{References}

Acker A., et al., 1992, Strasbourg-ESO Catalogue of Galactic Planetary Nebulae, ESO, Garching

Acker A., et al., 1996, A\&A 305, 944

Clegg R.E.S., et al., 1997, MNRAS 284, 348

Decin L., et al., 1998, A\&A 332, 928

Hrivnak B.J., 1995, ApJ 438, 349

Huggins P.J., 1996, A\&A 315284

Jura M., 1991, ApJ 372, 208

Jura M., Kleinmann S.G., 1989, ApJ 341, 359

Justtanont K., et al., 1996, A\&A 309, 612

Kastner J.H., 1996, ApJ 462, 777

Kholtygin A.F., 1998, A\&A 329, 691

Kingsburgh R.L., Barlow M.J., 1994, MNRAS 271, 257

Kwok S., 1993, ARA\&A 31, 63

Leisy P., Dennefeld M., 1996, A\&A Suppl. 116, 95

Omont A., et al., 1993, A\&A 267, 515

Parthasarathy M., 1993a, in "Luminous High Latitude Stars", ed. D.D. Sasselov, ASP Conf. Ser. 45, 173

Parthasarathy M., 1993b, ApJ 414, L109

Parthasarathy M., et al., 1993, A\&A 267, L19

Parthasarathy M., 1994, in "The MK process at 50 years", ed. C.J. Corbally et al., ASP Conf. Ser. 60, 261

Parthasarathy M., et al., 1995, A\&A 300, L25

Parthasarathy M., Acker A., Stenholm B., 1998, A\&A 329, L9

Parthasarathy M., Garcia-Lario P., Pottasch S.R., 1992, A\&A 264, 159

Parthasarathy M., Pottasch S.R., 1986, A\&A 154, L16

Peimbert M., 1978, IAU Symposium 76, ed. Y. Terzian, Reidel, p. 215

Pena M., Ruiz M.T., Torres-Peimbert S., 1997, A\&A 324, 674

Reddy B.E., Parthasarathy M., Gonzalez G., Bakker E.J., 1997, A\&A 328, 331

Rola C., Stasinska G., 1994, A\&A 282, 199

Tylenda R., Acker A., Stenholm B., 1993, A\&A Suppl. 102, 595

Van Winckel H., 1997, A\&A 319, 561

Waters L.B.F.M., et al., 1998, A\&A 331, L61

Webster A., 1995, MNRAS 277, 1555

Začs L., et al., 1995, MNRAS 275, 764

Zuckerman B., et al., 1976, ApJ 205, L15

Zuckerman B., Aller L.M., 1986, ApJ 301, 772 